\title{
REVIEW
}

\section{Liver natural killer cells: subsets and roles in liver immunity}

\author{
Hui Peng ${ }^{1,2}$, Eddie Wisse ${ }^{3}$ and Zhigang Tian ${ }^{1,2}$
}

The liver represents a frontline immune organ that is constantly exposed to a variety of gut-derived antigens as a result of its unique location and blood supply. With a predominant role in innate immunity, the liver is enriched with various innate immune cells, among which natural killer (NK) cells play important roles in host defense and in maintaining immune balance. Hepatic NK cells were first described as 'pit cells' in the rat liver in the 1970s. Recent studies of NK cells in mouse and human livers have shown that two distinct NK cell subsets, liver-resident NK cells and conventional NK (cNK) cells, are present in this organ. Here, we review liver NK cell subsets in different species, revisiting rat hepatic pit cells and highlighting recent progress related to resident NK cells in mouse and human livers, and also discuss the dual roles of NK cells in liver immunity.

Cellular \& Molecular Immunology (2016) 13,328-336; doi:10.1038/cmi.2015.96; published online 7 December 2015

Keywords: conventional NK cell; liver; liver-resident NK cell; pit cell; tolerance.

\section{INTRODUCTION}

The liver is an essential and complex organ that fulfills a wide range of functions to maintain the body's homeostasis. The liver is well known for its central role in many metabolic processes, including bile production, protein synthesis, fat metabolism, and glucose homeostasis. In recent years, accumulating studies have highlighted the importance of the liver in immune function. ${ }^{1,2}$ Because the liver is continually exposed to a large number of antigens derived from the gastrointestinal tract, ${ }^{3,4}$ the local immune system must constantly provide secure mechanisms to eliminate pathogens and toxins and to maintain tolerance to harmless dietary antigens. Excessive or otherwise inappropriate immune responses can lead to autoimmune liver diseases or chronic infections. ${ }^{5}$

As a component of the innate immune system, natural killer (NK) cells serve as a crucial first line of defense against invading tumor cells by mediating cytotoxicity and producing cytokines. ${ }^{6,7}$ Increasing evidence of cross talk between NK cells and other immune cells has implicated that NK cells also play an important role in shaping the adaptive immune response. ${ }^{8,9} \mathrm{NK}$ cells are derived primarily from the bone marrow and are widely distributed throughout the body in both lymphoid and non-lymphoid tissues. ${ }^{10,11}$ Recent studies have shown that NK cells in different tissues exhibit distinct phenotypic and functional characteris- tics, ${ }^{12,13}$ suggestive of a complex interplay between NK cell subset composition and the tissue environment.

The liver is considered a unique immunological organ with a predominantly innate immunity role, as it contains an unusually large number of innate immune cells and is particularly enriched with NK cells. ${ }^{14}$ Moreover, it has been reported for years that hepatic NK cells differ from peripheral NK cells in terms of surface marker expression, cytokine profiles, and cytotoxic potential. ${ }^{15}$ More recently, a unique NK cell subset, termed 'liver-resident NK cells,' was characterized in both mouse and human livers. ${ }^{16,17}$ In addition, in rat liver, subsets of NK cells were defined (see later). The relative abundance and unique features of hepatic NK cells have profound implications for the important function of these cells in this organ. Over the last decade, accumulating evidence has suggested the involvement of NK cells in the pathogenesis of liver diseases. ${ }^{18,19}$ Here, we highlight recent advances related to liver NK cell subsets and also review our current knowledge of NK cell functions in acute and chronic liver diseases.

\section{ANATOMICAL AND IMMUNOLOGICAL FEATURES OF THE LIVER}

The liver is the largest internal organ and lies under the diaphragm in the upper right quadrant of the abdominal cavity.

\footnotetext{
${ }^{1}$ Institute of Immunology and The CAS Key Laboratory of Innate Immunity and Chronic Disease, School of Life Sciences and Medical Center, University of Science and Technology of China, Hefei, Anhui, China; ${ }^{2}$ Collaborative Innovation Center for Diagnosis and Treatment of Infectious Diseases, State Key Laboratory for Diagnosis and Treatment of Infectious Diseases, First Affiliated Hospital, College of Medicine, Zhejiang University, Hangzhou, Zhejiang, China and ${ }^{3}$ Maastricht Multimodal Molecular Imaging Institute, Division of Nanoscopy, Universiteitssingel 50, Maastricht, The Netherlands

Correspondence: E Wisse: Maastricht Multimodal Molecular Imaging institute, Division of Nanoscopy, Universiteitssingel 50, 6229 ER Maastricht, The Netherlands (eddie.wisse@telenet.be); Z Tian: School of Life Sciences, University of Science and Technology of China, 443 Huang-Shan Road, Hefei, Anhui 230027, China. (tzg@ustc.edu.cn)
}

Received: 3 August 2015; Revised: 14 October 2015; Accepted: 19 October 2015 
Notably, the liver has a dual blood supply, with approximately $80 \%$ of its blood supplied from the gastrointestinal tract via the portal vein and the remaining $20 \%$, which is rich in oxygen, supplied from the hepatic artery. ${ }^{20}$ The portal veins and hepatic arteries terminate and branch together in the intrahepatic portal tract, and the blood leaves the liver parenchyma via the central hepatic veins after passing through an anastomosing network of sinusoids. ${ }^{21}$ The diameter of the hepatic sinusoids is narrow $(5-7 \mu \mathrm{m}),{ }^{22}$ and the blood pressure is much lower than in the systemic circulation, resulting in a relatively slow flow in the liver sinusoids. This reduced blood flow slows the passage of gut-derived antigens, facilitating the clearance of harmful substances by liver-resident cells and maximizing the interaction between circulating lymphocytes and the hepatic sinusoidal endothelium. ${ }^{20}$

The liver is composed of different cell types with distinct functions. Hepatocytes, which are the liver parenchymal cells, account for approximately two-thirds of the total cell population. ${ }^{2}$ Hepatocytes communicate with the blood plasma and its constituents through open fenestrae with diameters on the order of $105 \mathrm{~nm}$ (human liver) to $140 \mathrm{~nm}$ (murine liver). ${ }^{23}$ Non-parenchymal cells are located primarily in the sinusoidal compartment of the tissue, including sinusoidal endothelial cells $^{24}$ Kupffer cells, ${ }^{25}$ lymphocytes, and myeloid cells. ${ }^{26}$ Hepatocytes are separated from the endothelial cells by the space of Disse, which contains hepatic stellate cells (HSCs) and extracellular matrix proteins. ${ }^{21}$ Interestingly, among the non-parenchymal cells, there are several cell populations endowed with antigen-presenting potential, which contributes to immunological tolerance under non-inflammatory conditions. ${ }^{27}$ For example, antigen-specific interactions with $\mathrm{CD} 8{ }^{+}$ $\mathrm{T}$ cells increase the expression of the co-inhibitory B7-H1 molecule on liver sinusoidal endothelial cells, which can induce $\mathrm{CD}^{+} \mathrm{T}$ cell tolerance via interaction with programmed cell death protein 1 expressed on $\mathrm{CD}^{+}{ }^{+}$cells. $^{28}$

As a lymphoid organ, the liver also contains a diverse population of innate lymphocytes, such as NK cells, natural killer T (NKT) cells, and $\gamma \delta$ T cells, and adaptive lymphocytes, such as $\alpha \beta T$ cells and B cells. In particular, intrahepatic lymphocytes have a unique subset composition, with a notable enrichment in innate immune cells. In humans, up to $65 \%$ of all hepatic lymphocytes consist of NK cells, NKT cells, and $\gamma \delta$ T cells ${ }^{29}$; these cell populations can proliferate under certain experimental or pathological conditions. The dominating presence of these populations in the liver and in early defense against pathogens places these cells in a key position among effector lymphocytes in liver immune surveillance.

\section{WHAT IS AN NK CELL?}

NK cells were first identified in the 1970s based on their natural ability to kill tumor cells in the absence of prior stimulation. ${ }^{30,31}$ Beyond their role as cytolytic effector lymphocytes, NK cells are also considered major producers of certain cytokines, such as IFN- $\gamma$, through which NK cells can contribute to shaping the adaptive immune response. ${ }^{9,32}$ Although NK cells have been classified as innate immune cells due to their lack of antigen-specific receptors, several lines of evidence have revealed that NK cells can mediate antigen-specific immunological memory, challenging the conventional distinction between innate and adaptive immunity. ${ }^{33}$

NK cell effector functions are controlled by a variety of cell surface activating and inhibitory receptors. The activating receptors expressed by NK cells primarily include the natural cytotoxicity receptors (NKp46, NKp44, and NKp30), CD16, and Ctype lectin receptors (CD94/NKG2C, NKG2D, NKG2E/H, and NKG2F). ${ }^{6}$ Some activating receptor ligands, e.g., NKG2D ligands (the human UL16-binding protein and major histocompatibility complex (MHC) class I chain-related molecules and the mouse RAE1, H60, and MULT1 molecules), are inducible in conditions of cellular stress, rendering the stressed cell susceptible to lysis by NK cells. ${ }^{34} \mathrm{NK}$ cells are equipped with an array of MHC class I-specific inhibitory receptors, including the killer cell immunoglobulin-like receptors in humans, the lectin-like Ly49 dimers in mice and the lectin-like CD94-NKG2A heterodimers in both species. ${ }^{35}$ In general, healthy cells constitutively express MHC class I, which binds to NK cell inhibitory receptors, allowing the cell to avoid NK cell attack. However, cells may downregulate MHC class I expression in response to stress ('missing-self), such as viral infection or cellular transformation, and can then be attacked by NK cells because activating signals dominate over inhibitory signals. ${ }^{36}$

NK cells are derived primarily from the bone marrow, in which NK cells develop in a stepwise manner in association with the progressive acquisition and/or downregulation of a series of markers and functional maturation. ${ }^{10,37}$ However, several lines of evidence have indicated the presence of hematopoietic progenitors and immature NK cells at extramedullary sites, ${ }^{16,38}$ as well as NK cell diversity in distinct tissues. ${ }^{39}$ For example, NK cells can originate in the thymus and differ from bone marrow-derived NK cells by expressing more GATA-3 and CD127 (interleukin $7 \mathrm{R} \alpha, \mathrm{IL}-7 \mathrm{R} \alpha$ ) and requiring IL-7 signals for their development, ${ }^{40}$ suggesting that NK cell development may take place at extramedullary sites, where local environmental signals can affect this process and result in the development of distinct NK cell subsets. ${ }^{41}$

\section{LIVER NK CELL SUBSETS}

The discovery of a new cell type in a well-studied organ such as the liver is a thrilling experience. This event occurred during a microscopic study of rat liver, after perfusing the portal vein with a buffered and isotonic glutaraldehyde solution. ${ }^{42}$ By applying this technique, 'pit cells,' which are now known to be the NK cells of rat liver, were discovered the year after the initial identification of NK cells; this report was the first morphological (electron microscopic, EM) description of liver NK cells. Although most previous studies of NK cells focused on mouse splenic and human peripheral blood NK cells, which are circulating and referred to as conventional NK (cNK) cells, ${ }^{43}$ with the recent boom of organ-specific NK cells, our knowledge of liver NK cell biology has been greatly expanded. 


\section{Pit cells: the NK cells of rat liver}

Within this context, the pit cell presented itself. ${ }^{42}$ We found this cell to be different from the visiting blood cells that are typically seen in a healthy rat liver sinusoid, i.e., granulocytes (polymorphonuclear, eosinophils, and basophils), lymphocytes, monocytes, and occasional plasma cells. However, the relatively frequent occurrence of these unknown cells in the normal rat liver prompted us to determine whether we were dealing with a new type of resident cell. To make a discussion possible, we needed a name, and we applied the name 'pit cell,' which was inspired by the presence of a number of small granules, as is the case with the little seeds in grapes for which the Dutch word 'pit' is used.

Pit cells have a diameter between those of lymphocytes and granulocytes. Similar to Kupffer cells, the shape of pit cells is irregular and variable (Figure 1). Pit cells are attached to the endothelial lining, which they might penetrate with little microvilli 'shaking hands' with the microvilli of parenchymal cells, as if they are anchoring or securing their position. Pit cells are not located in the space of Disse. These cells are often neighbors of Kupffer cells and even make contact with Kupffer cells, suggesting some type of physical relationship. Most remarkable are the numerous, characteristic granules $(\varnothing 0.3 \mu \mathrm{m})$ that lie together in one part of the cytoplasm (Figure 2). These granules are round and have an electrondense content with an even darker characteristic dense core. This content is surrounded by an electron-lucent halo and a single membrane. The other part of the cytoplasm is hyaloplasmic or contains only a few small organelles, such as single strands of rough endoplasmic reticulum, a small Golgi apparatus, free ribosomes, and few small mitochondria. Kaneda et al. ${ }^{44}$ described an additional type of granule for pit cells: the so-called 'rod cored vesicle.' These granules are smaller, less frequent, and electron-lucent with a central rod, and they are not comparable to the typical pit cell granules. We also found pit cells with fewer and larger granules in the peripheral blood and the spleen. It was easy to find pit cells in the livers of other species (dogs, mice, and hamsters), except for human liver. Unlike Kupffer cells, pit cells do not possess a phagocytic or endocytic function. Pit cells were unreactive in many experimental conditions, except for partial hepatectomy, in which we found pit cell mitosis in the remaining part of the liver. ${ }^{42}$

The first possible function of pit cells was suggested by Kaneda et al., ${ }^{44}$ who noted that pit cells show some morphological similarity to large granular lymphocytes (LGLs). LGLs are known for their larger than lymphocyte size and their azurophilic granules. After isolating LGLs from the peripheral blood of rats, the capacity of these cells to spontaneously kill YAC-1 tumor cells was demonstrated.

To further analyze the functional characteristics of pit cells, a good method for the isolation and purification of these cells from rat liver was needed. A simple and effective method of sinusoidal lavage was developed by Bouwens et al. ${ }^{45}$ The authors were able to wash pit cells out of the liver by flushing the portal vein at a water pressure of $50 \mathrm{~cm}$ (physiological saline) without the use of enzymes. These cells were found to be highly cytotoxic against YAC4 lymphoma tumor cells, and this reaction could be blocked by an antibody against NK cells (anti-asialo GM1). In these preparations, it was demonstrated that the specific granules of the cells were acid phosphatase-

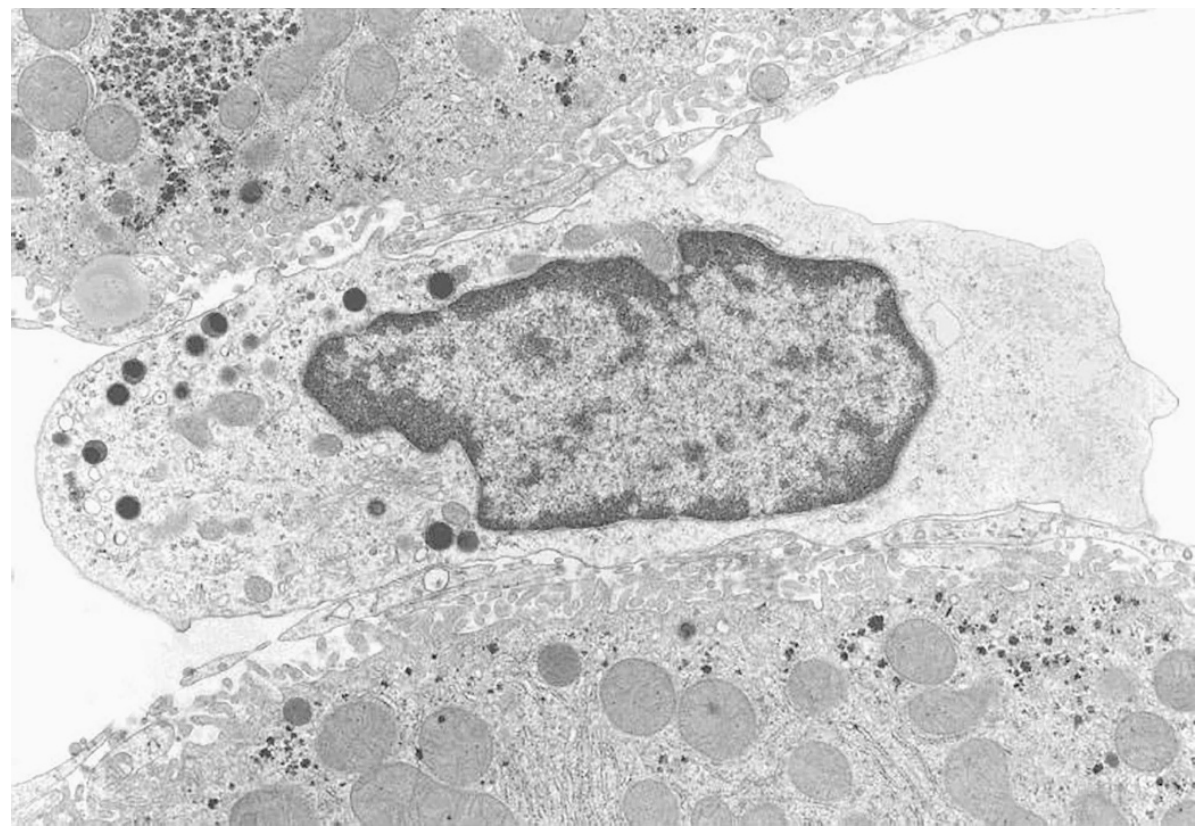

Figure 1 Pit cell in a normal rat liver (Wistar). Organelles are situated on one side of the central nucleus. The other side of the cell is composed of hyaloplasm without organelles. The pit cell makes contact with sinusoidal endothelial cells, and microvilli sometimes protrude into the space of Disse (top left). Pit cells possess characteristic electron-dense granules, in addition to a small number of mitochondria, RER cisternae, and a Golgi apparatus. The pit cell in this figure measures approximately $8 \mu \mathrm{m}$ in length. 


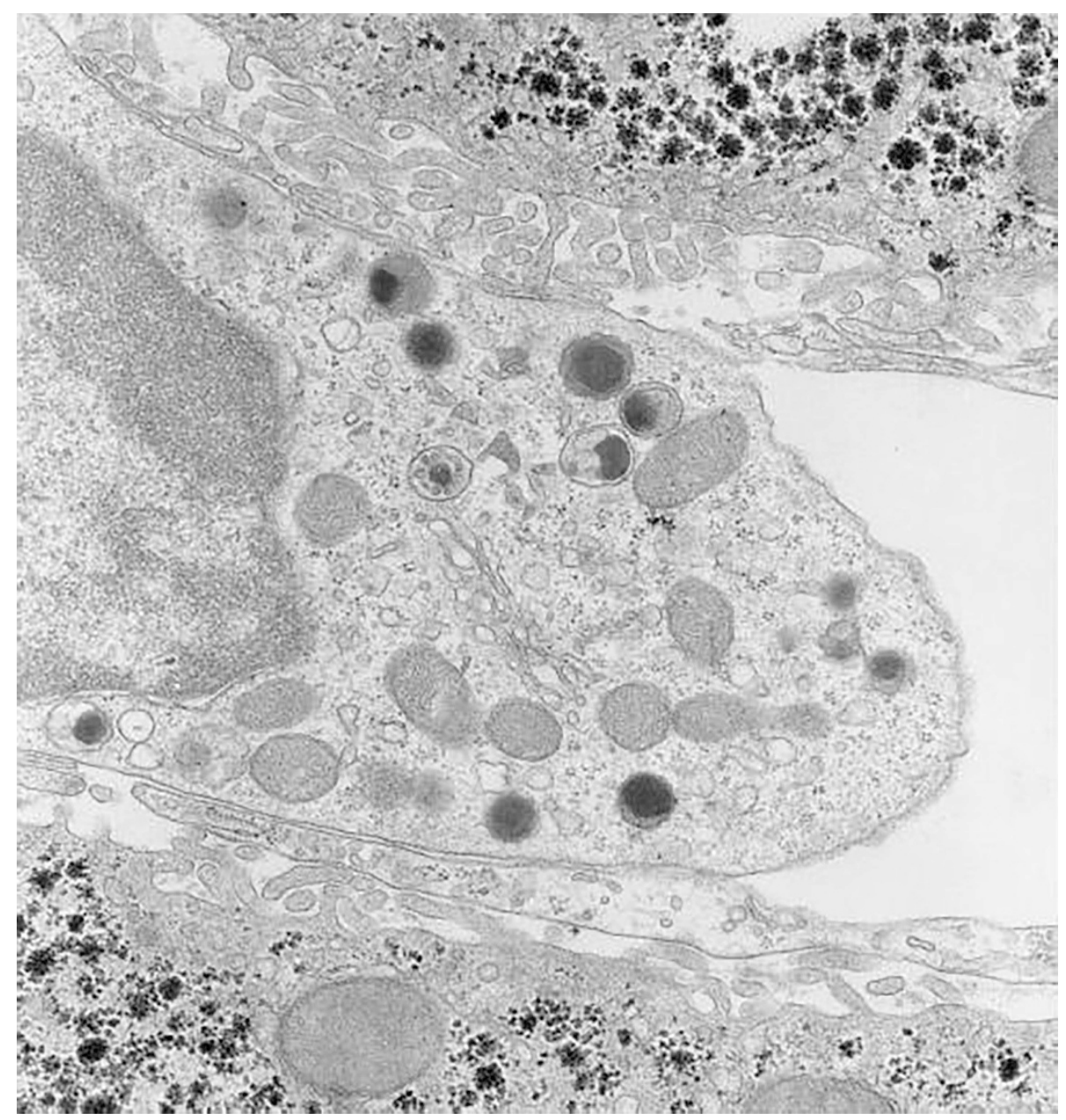

Figure 2 Part of the cytoplasm of a rat liver pit cell at higher magnification, showing the typical structure of the granules. Other organelles in this picture are rod-cored vesicles (smaller than the dense granules and without dense content), mitochondria, a Golgi apparatus, and free ribosomes. A microvesicular body is situated above the Golgi apparatus. The pit cell makes contact with the fenestrated sinusoidal endothelial lining that covers the microvilli of the parenchymal cells, together forming the space of Disse. Original magnification, approximately $\times 10000$.

positive, indicating that they are lysosomes. The cells could be further purified and enriched by applying a low-density (LD) Percoll gradient.

Cells of the reticuloendothelial system, such as Kupffer cells, can be functionally activated and increased in number by biological response modifiers, including zymosan, Propionobacterium acnes and OK-432. Indeed, the application of these substances increases the number of Kupffer cells considerably; however, pit cells were increased even more (sixfold) by the same stimuli. Irradiation and metaphase arrest demonstrated that the increased number of pit cells was the result of local, hepatic proliferation. ${ }^{46}$ IL-2 had an even stronger effect on pit cell proliferation. ${ }^{47}$ In addition, the parallels between the reactions of Kupffer cells and pit cells are obvious. When Kupffer cells were eliminated from the liver by treatment with dichloromethylene diphosphonate, the number of pit cells was also reduced. In vitro, pit cells showed signs of activation after the addition of Kupffer cell-conditioned medium. ${ }^{48}$ Therefore, Kupffer cell and pit cell numbers and functions seem to rise and fall together.
The presence of pit cells inside and outside rat liver stimulated further studies of the possible relationships between these cells. Pit cells that were isolated via sinusoidal lavage were separated into LD and high-density (HD) fractions using a $45 \%$ Percoll gradient. When these cells were compared with blood LGLs, within the order LGL $>$ HD $>$ LD, the LD pit cells were eight times more cytotoxic than blood NK cells against a number of different tumor cell lines. ${ }^{49}$ Based on these and other data, it may be concluded that peripheral blood LGLs are able to take up residence in the liver as HD pit cells and subsequently develop into LD pit cells with increased tumoricidal potential, possibly under the influence of Kupffer cells. ${ }^{49}$

Rat liver pit cells can be immunostained with the monoclonal antibody 3.2.3. Using this staining technique, it was possible to quantify the number of pit cells in liver frozen sections as $13.7 \pm 1.1 / \mathrm{mm}^{2}{ }^{50}$ The lobular distribution of pit cells in the liver was found to be predominantly $(\sim 60 \%)$ periportal. Again, this distribution coincides with the location of Kupffer cells. 
When syngeneic CC531 colon carcinoma cells are killed, the cytotoxicity of rat liver pit cells seems to be based on the induction of apoptosis via the perforin/granzyme pathway rather than the Fas pathway (Figure 3). ${ }^{51}$

In recent years, the author (E. W.) had the opportunity to investigate more than 200 wedge and needle biopsies of human livers using fixation methods adapted to obtain perfusion fixation quality tissue. ${ }^{52,53}$ After observing these specimens, the author concluded that no cells with rat pit cell morphology are present in the human liver. Very occasionally, a cell with a few granules could be found, but an EM comparison of rat and human livers led to the conclusion that human liver does not harbor a morphological equivalent of the rat pit cell.

\section{Liver-resident NK cells}

In mice, liver NK cells are present at significantly higher frequencies than NK cells in the bone marrow, peripheral blood, and spleen, accounting for approximately $5-10 \%$ of the total lymphocytes present in this tissue. ${ }^{54,55}$ More than 10 years ago, Kim et al. unexpectedly observed the presence of a high frequency of phenotypically immature NK cells in the murine liver; ${ }^{37}$ these cells express low amounts of DX5, Mac-1, and Ly49 receptors, which are the markers associated with NK cell maturation. ${ }^{37,56}$ Subsequently, the phenotype and function of liver NK cells were further studied in detail ${ }^{15}$; the phenotypically immature NK cells in the liver have been reported to express high levels of the effector molecule TRAIL, with cytotoxicity against tumor cells. TRAIL $^{+} \mathrm{NK}$ cells predominate in fetal and neonatal mice and persist in the liver, but not the spleen, until adulthood. ${ }^{15}$ Over that period, these unique hepatic NK cells were still considered to be an intermediate stage during the development of mature cNK cells.

Recently, we found that the mutually exclusive expression of CD49a and DX5 can distinctively divide mouse liver NK cells into two subsets, CD49a ${ }^{-} \mathrm{DX} 5^{+}$and $\mathrm{CD} 49 \mathrm{a}^{+} \mathrm{DX} 5^{-},{ }^{16}$ and substantial differences exist between these two subsets (Figure 4). In contrast to the $\mathrm{CD} 49 \mathrm{a}^{-} \mathrm{DX} 5^{+}$subset, $\mathrm{CD} 49 \mathrm{a}^{+} \mathrm{DX} 5^{-} \mathrm{NK}$ cells are rarely found in the bone marrow, peripheral blood, and spleen. Of particular interest, $\mathrm{CD} 49 \mathrm{a}^{+} \mathrm{DX} 5^{-} \mathrm{NK}$ cells selectively reside in the liver sinusoid blood, but are not present in the afferent and efferent blood of the liver. Parabiosis studies further confirmed that the cells of the CD49a ${ }^{+} \mathrm{DX} 5^{-}$subset rarely

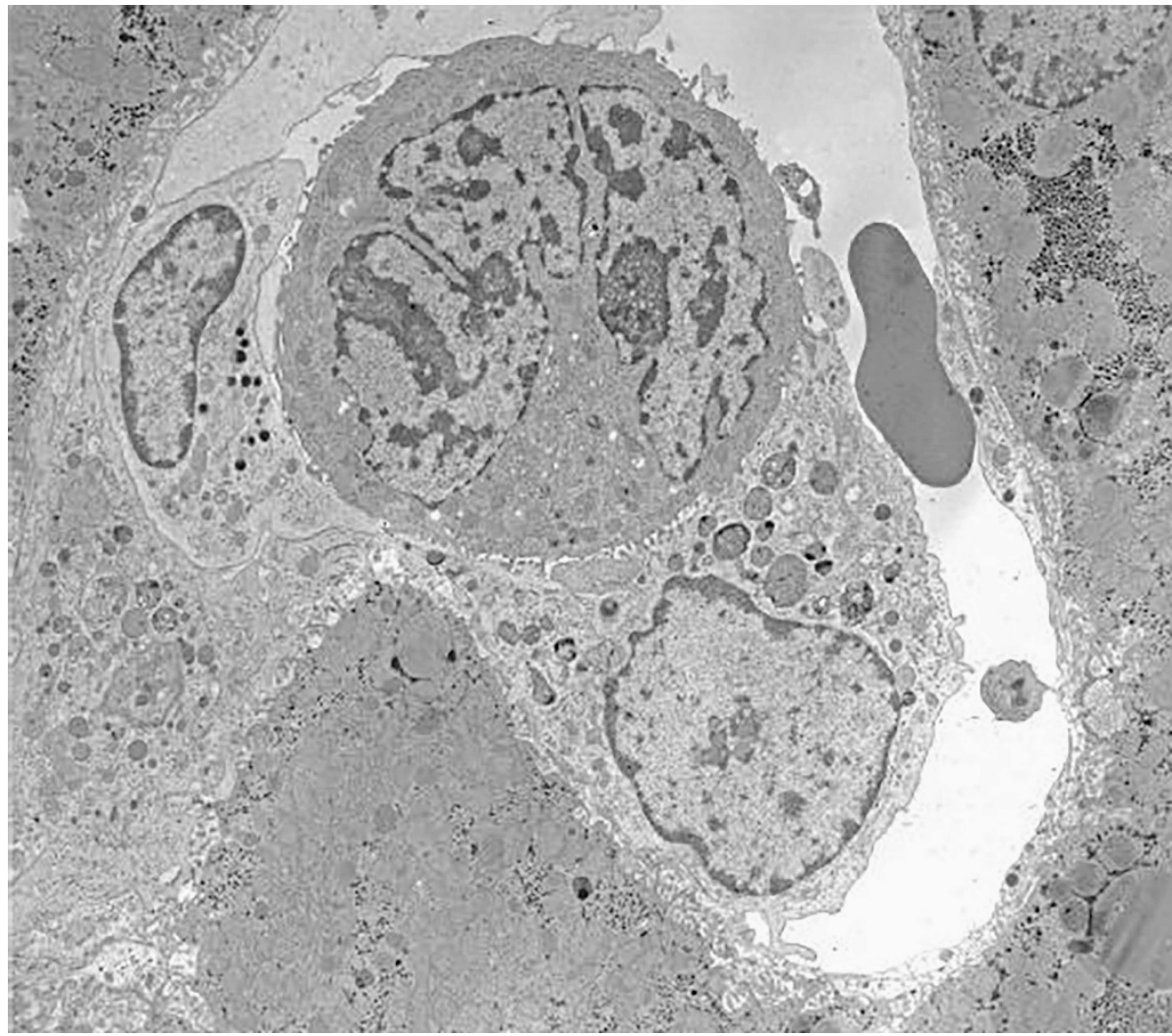

Figure 3 One hour after the injection of colon carcinoma cells (CC531) into syngeneic Wagrain rats, a pit cell (left) and a Kupffer cell (lower right) were found attached to a tumor cell (center). Pit cells kill these tumor cells in vitro by secreting perforin and granzyme, causing the apoptosis of the tumor cells. Note that the pit cell granules are assembled at the side facing the tumor cell. The pit cell has not degranulated. It is thought that this secretion triggers membrane changes in the tumor cells, which are recognized by the Kupffer cell and initiate the phagocytic reaction. The first stage in phagocytosis is determined by the attachment of the effector cell to the target cell, which is clearly depicted here. The combination of apoptotic induction (by the pit cell) and phagocytosis (by the Kupffer cell) will kill this tumor cell. The single red blood cell in the sinusoid measures approximately $7 \mu \mathrm{m}$. 


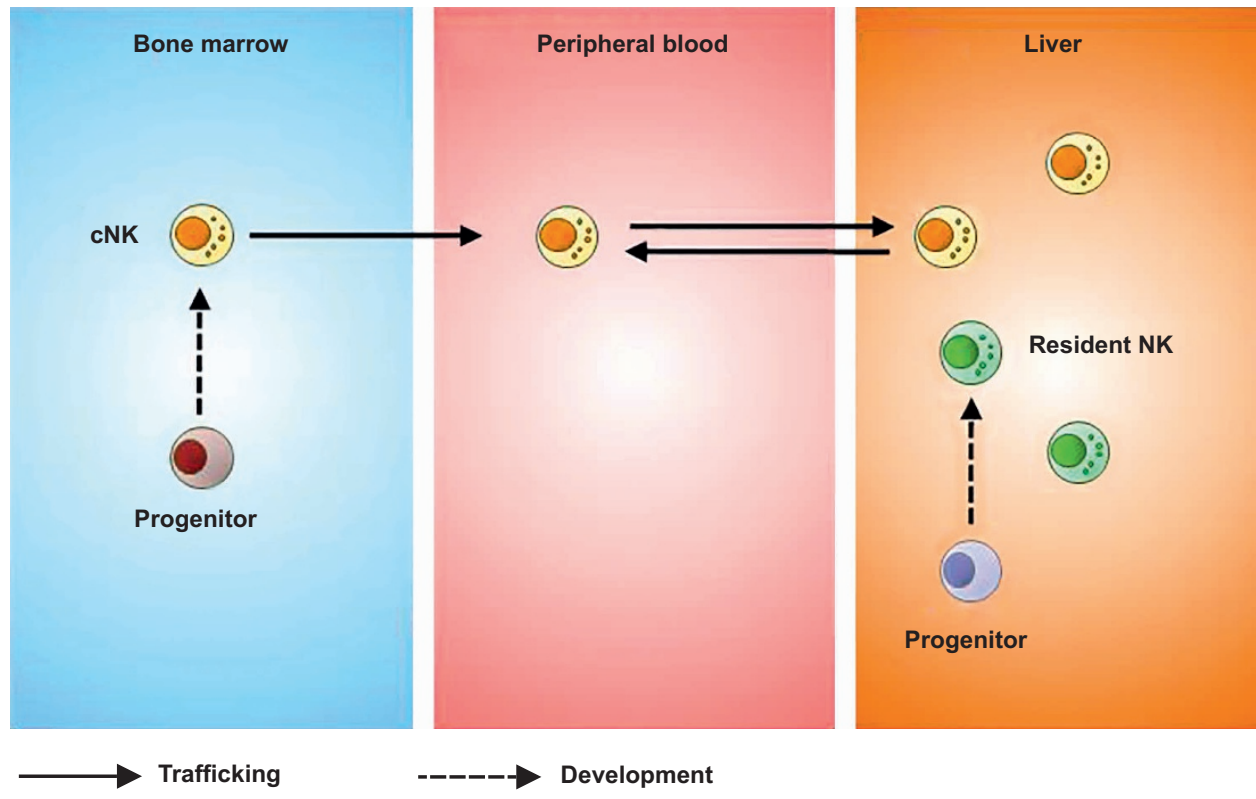

Figure 4 The adult liver contains two NK cell subsets, cNK cells, and tissue-resident NK cells, the latter of which can develop from liver hematopoietic progenitor cells.

circulate or emigrate from the liver, whereas $\mathrm{CD} 49 \mathrm{a}^{-} \mathrm{DX} 5^{+}$ cells are highly migratory under homeostatic conditions. ${ }^{16}$ For these reasons, the hepatic $\mathrm{CD} 49 \mathrm{a}^{+} \mathrm{DX} 5^{-}$subset is termed 'liver-resident NK cells,' whereas the $\mathrm{CD} 4 \mathrm{a}^{-} \mathrm{DX} 5^{+}$subset represents cNK cells. More recently, it was found that the CD $49 \mathrm{a}^{+}$DX $5^{-}$phenotype can also be used to identify tissueresident NK cells in the uterus and skin. ${ }^{39}$ Liver-resident NK cells are more efficient in secreting a broad pattern of cytokines and chemokines, including interferon gamma (IFN- $\gamma$ ), tumor necrosis factor- $\alpha$ (TNF- $\alpha$ ), ganulocyte-macrophage colonystimulating factor, and chemokine (C-C motif) ligand 3 , in comparison to cNK cells, which primarily produce IFN$\gamma \cdot{ }^{39,57}$ These two NK cell subsets express a distinct repertoire of cytotoxic effector molecules, and, compared with cNK cells, liver-resident NK cells degranulate poorly in response to certain cell lines, as measured by surface CD107a. ${ }^{16,39}$ Further studies are warranted to compare the cytotoxicity of these two NK cell subsets in detail.

Although liver-resident NK cells resemble immature cNK cells in phenotype, adoptive transfer studies showed that these cells preferentially home to the liver and do not convert to $\mathrm{DX}^{+}$cNK cells, suggesting that liver-resident NK cells are stable under steady-state circumstances and may develop separately from cNK cells. ${ }^{16}$ Further support for this notion came from the observation that liver-resident NK cells and cNK cells have different transcription factor requirements for their development. For example, liver-resident NK cells are Eomes negative, and their development is strictly dependent on T-bet but not on Eomes ${ }^{54,57}$; conversely, cNK cells are present in T-bet-deficient mice and express high levels of Eomes, which is essential for their development. ${ }^{54}$ Additionally, $\mathrm{PLZF}^{+}$or $\mathrm{Id} 2^{+}$progenitors have been shown to have the potential to develop into liver-resident NK cells and other innate lymphoid cells, but not cNK cells. This finding indicates the existence of two alternative pathways for the development of liver-resident NK cells and cNK cells. Additionally, liver-resident NK cells are distinct from thymic NK cells, as they develop independent of the thymus or GATA- $3 .^{39}$ Regarding the origin of liverresident NK cells, our studies show that hematopoietic progenitor cells residing in the adult liver can give rise to liver-resident NK cells, ${ }^{16}$ raising the possibility that an intrahepatic developmental pathway may exist for liver-resident NK cells.

Several findings suggested that NK cells can mediate memory-like responses after specific activation by chemical haptens ${ }^{58-60}$ or viruses ${ }^{59,61,62}$ or non-specific activation by cytokines. ${ }^{63}$ In particular, in a hapten-induced contact hypersensitivity (CHS) model, antigen-specific memory NK cells were found to reside in the livers of hapten-sensitized mice rather than in the spleen; these findings prompted us to investigate whether liver-resident NK cells contributed to haptenspecific CHS responses. ${ }^{16}$ Using the adoptive transfer of hepatic NK cell subsets from hapten-sensitized mice into naive mice, our study revealed that liver-resident NK cells appeared to mediate a recall response in recipients after challenge with the same hapten, whereas cNK cells failed to mediate such a response.

In humans, the liver also contains a unique $\mathrm{CD} 49 \mathrm{a}^{+} \mathrm{NK}$ cell subset that resembles murine liver-resident NK cells. ${ }^{17}$ Similar to their counterparts in the mouse liver, human $\mathrm{CD} 49 \mathrm{a}^{+} \mathrm{NK}$ cells are T-bet ${ }^{+}$Eomes $^{-}$and are not detectable in afferent or efferent hepatic venous or peripheral blood. Moreover, these cells produce high levels of proinflammatory cytokines but degranulate poorly in comparison to human $\mathrm{CD} 49 \mathrm{a}^{-} \mathrm{cNK}$ cells. Therefore, CD49a expression can also be used to characterize human liver-resident NK cells. 


\section{LIVER NK CELLS IN HEPATIC IMMUNITY AND TOLERANCE}

The liver has long been recognized as an immune-tolerant organ, as liver allografts are less likely to be rejected than other transplanted organs. ${ }^{64}$ Moreover, liver allografts lead to donorspecific tolerance that can further facilitate the acceptance of other donor-originated organs, such as heart or kidney allografts, without the need for immunosuppressive agents. ${ }^{65}$ In addition, hepatic tolerance also refers to the fact that the liver is tolerant to a large number of dietary and commensal antigens under homeostatic conditions. The tolerogenic properties and special anatomy of the liver predispose this organ to pathogen invasion and sustain persistent infection. Indeed, several common pathogens, such as hepatitis $B$ virus (HBV) and hepatitis $C$ virus (HCV), selectively target the liver and establish chronic infections. ${ }^{66}$ Various types of hepatic cells are involved in the maintenance of immunotolerance, and NK cells appear to play a dual role in this process.

Given their ability to secrete proinflammatory cytokines and mediate cytotoxicity, it is not surprising that NK cells can induce liver immunity toward an immunogenic status. It has been reported that polyinosinic/polycytidylic acid (poly I:C), which is a synthetic viral RNA mimic, induces NK cell activation and accumulation in the liver, leading to mild hepatitis. ${ }^{67}$ In a murine fulminant hepatitis model established by the injection of poly I:C and D-galactosamine, the interaction between liver NK cells and Kupffer cells via NKG2D-Rael recognition induces the production of IFN- $\gamma$ and TNF- $\alpha$ and results in severe liver injury. ${ }^{68}$ Murine hepatitis virus-induced NK cell activation also plays a critical role in the development of hepatocyte necrosis and fulminant hepatic failure. ${ }^{69}$

NK cells promote hepatic tolerance. It has been reported that NK cells can inhibit dendritic cell (DC) activation by producing the suppressive factors transforming growth factor-beta (TGF- $\beta$ ) and IL-10 in an NKG2A-dependent manner. The resulting tolerogenic DCs further induce the expansion of regulatory $\mathrm{T}$ cells, contributing to the induction of an immunotolerant state during $\mathrm{HCV}$ infection. ${ }^{70,71}$ Accumulating evidence demonstrated that NK cell function is compromised during chronic hepatic infection, which consequently contributes to pathogen persistence. For instance, NK cells from HBV patients or HBV carrier mice exhibit increased levels of NKG2A, which are responsible for reduced NK cell cytotoxicity and viral persistence. ${ }^{72}$ Additionally, chronic HBV infection induces high levels of soluble TGF- $\beta 1$, which in turn reduce the expression of the activating receptors NKG2D and 2B4 on NK cells and impair their effector functions. ${ }^{73}$ In a steady-state situation, the mouse liver contains a population of functionally hyporesponsive NK cells that express NKG2A and lack Ly49 receptor expression. The enrichment of these cells in the liver is partially attributed to the high levels of IL-10 that are present within this organ. ${ }^{74}$ Therefore, the immunosuppressive environment plays a role in shaping liver NK cells, through which hepatic tolerance can be further consolidated.
NK cells also play important roles in other liver diseases, such as liver fibrosis and liver regeneration. ${ }^{75,76}$ The anti-fibrotic effect of NK cells, which is accomplished by the killing of activated HSCs, was first observed in experimental mouse models in $2006,{ }^{77,78}$ and similar findings were subsequently obtained in patients. ${ }^{79-81}$ Clinical studies demonstrated that activated HSCs induce NK cell degranulation/activation, which in turn promotes hepatic stallete cell apoptosis in a TRAIL-, FasL-, and NKG2D-dependent manner. ${ }^{81} \mathrm{~A}$ recent study found that NK cell activity against HSCs is inhibited by $\mathrm{CD}^{+}{ }^{+}$regulatory T cells (Tregs) from either healthy controls or $\mathrm{HCV}$ patients, ${ }^{82}$ suggesting that the anti-fibrotic activity of NK cells can be modified by crosstalk with other immune cells.

The liver has the unique ability to regenerate after tissue damage, and several lines of evidence have indicated that NK cells play a role in liver regeneration. Suppressing NK cell activity using the immunosuppressive drug FK506 or depleting NK cells using anti-asialo GM1 treatment promotes liver regeneration, ${ }^{83,84}$ whereas the poly I:C- or viral infection-induced activation of NK cells negatively regulates liver regeneration in an IFN- $\gamma$-dependent manner. ${ }^{84}$ Recently, the co-inhibitory receptor T cell Ig and ITIM domain was found to maintain NK cell tolerance by inhibiting the activation and IFN- $\gamma$ production of these cells, which ultimately protects liver regeneration. $^{85}$

\section{CONCLUDING REMARKS}

In recent years, it has been increasingly appreciated that the liver functions as an important innate immune organ. The unique anatomy and blood supply of this organ serve as an interface between the host and the external environment. Accumulating evidence demonstrated that NK cells constitute an abundant subfraction of hepatic lymphocytes that play important roles in host defense and immune balance in the liver. With the expansion of our knowledge of NK cell biology during the last few years, the unique characteristics of distinct subsets of liver NK cells have gradually been brought to light.

In summary, liver NK cells were first described as pit cells, which are easily characterized based on EM morphology in rat liver. These cells possess numerous characteristic granules, normal organelles, and rod-cored vesicles. Pit cells bear their granules on one side of the nucleus and attach to the sinusoidal endothelial lining. The cells often sit next to and make contact with Kupffer cells. Their numbers and activity levels increase and decrease together with the number and activity of Kupffer cells. This observation appears to indicate that a symbiosis or synergism exists between these two cell types. Pit cells have a high spontaneous cytotoxicity against a variety of tumor cells, which they kill using the perforin/granzyme pathway. In vivo, pit cells and Kupffer cells cooperate, with Kupffer cells phagocytosing tumor cells attacked by pit cells. Recently, liver-resident NK cells were characterized in both mice and humans. These cells are distinct from cNK cells in terms of phenotype, function, and development, but their relationship with pit cells awaits further study. Moreover, many studies of NK cells in liver diseases used bulk liver NK cells from rodents or human peripheral blood NK cells. Further studies using organ-specific 
NK cell subsets are needed to improve our understanding of the roles of liver-resident NK cells and cNK cells in liver function.

\section{COMPETING FINANCIAL INTERESTS}

The authors have declared that no competing interests exist.

\section{ACKNOWLEDGEMENTS}

This work was supported by the Ministry of Science \& Technology of China (973 Basic Science Project \#2013CB944902), the Natural Science Foundation of China (\#81361120388, \#31300727, and \#81571522), and the Key Program of the Chinese Academy of Sciences (KJZD-EW-L10 -003).

1 Crispe IN. The liver as a lymphoid organ. Annu Rev Immunol 2009; 27: 147-163.

2 Racanelli V, Rehermann B. The liver as an immunological organ. Hepatology 2006; 43: S54-62.

3 Berg RD. Bacterial translocation from the gastrointestinal tract. Trends Microbiol 1995; 3: 149-154.

4 Son G, Kremer M, Hines IN. Contribution of gut bacteria to liver pathobiology. Gastroenterol Res Pract 2010; 2010: 453563.

5 Hudspeth K, Pontarini E, Tentorio P, Cimino M, Donadon M, Torzilli G et al. The role of natural killer cells in autoimmune liver disease: a comprehensive review. J Autoimmun 2013; 46: 55-65.

6 Cheng M, Chen Y, Xiao W, Sun R, Tian Z. NK cell-based immunotherapy for malignant diseases. Cell Mol Immunol 2013; 10: 230-252.

7 Sun C, Sun H, Zhang C, Tian Z. NK cell receptor imbalance and NK cell dysfunction in HBV infection and hepatocellular carcinoma. Cell Mol Immunol 2015; 12: 292-302.

8 Vivier E, Raulet DH, Moretta A, Caligiuri MA, Zitvogel L, Lanier LL et al. Innate or adaptive immunity? The example of natural killer cells. Science 2011; 331: 44-49.

9 Crome SQ, Lang PA, Lang KS, Ohashi PS. Natural killer cells regulate diverse T cell responses. Trends Immunol 2013; 34: 342-349.

10 Di Santo JP. Natural killer cell developmental pathways: a question of balance. Annu Rev Immunol 2006; 24: 257-286.

11 Gregoire C, Chasson L, Luci C, Tomasello E, Geissmann F, Vivier E, Walzer $\mathrm{T}$ et al. The trafficking of natural killer cells. Immunol Rev 2007; 220: 169-182.

12 Shi FD, Ljunggren HG, La Cava A, Van Kaer L. Organ-specific features of natural killer cells. Nat Rev Immunol 2011; 11: 658-671.

$13 \mathrm{Yu}$ J, Freud AG, Caligiuri MA. Location and cellular stages of natural killer cell development. Trends Immunol 2013; 34: 573-582.

14 Gao B, Jeong WI, Tian Z. Liver: an organ with predominant innate immunity. Hepatology 2008; 47: 729-736.

15 Takeda K, Cretney E, Hayakawa Y, Ota T, Akiba H, Ogasawara K et al. TRAIL identifies immature natural killer cells in newborn mice and adult mouse liver. Blood 2005; 105: 2082-2089.

16 Peng H, Jiang X, Chen Y, Sojka DK, Wei H, Gao X et al. Liver-resident NK cells confer adaptive immunity in skin-contact inflammation. J Clin Invest 2013; 123: 1444-1456.

17 Marquardt N, Beziat V, Nystrom S, Hengst J, Ivarsson MA, Kekäläinen $\mathrm{E}$ et al. Cutting edge: identification and characterization of human intrahepatic CD49a+ NK cells. J Immunol 2015; 194: 2467-2471.

18 Tian Z, Chen Y, Gao B. Natural killer cells in liver disease. Hepatology 2013; 57: 1654-1662.

19 Jost S, Altfeld M. Control of human viral infections by natural killer cells. Annu Rev Immunol 2013; 31: 163-194.

20 Jenne CN, Kubes P. Immune surveillance by the liver. Nat Immunol 2013; 14: 996-1006.

21 Adams DH, Eksteen B. Aberrant homing of mucosal T cells and extraintestinal manifestations of inflammatory bowel disease. Nat Rev Immunol 2006; 6: 244-251.

22 MacPhee PJ, Schmidt EE, Groom AC. Intermittence of blood flow in liver sinusoids, studied by high-resolution in vivo microscopy. Am J Physiol 1995; 269: G692-698.

23 Wisse E, De Zanger RB, Charels K, Van Der Smissen P, McCuskey RS. The liver sieve: considerations concerning the structure and function of endothelial fenestrae, the sinusoidal wall and the space of Disse. Hepatology 1985; 5: 683-692.

24 Wisse E. An ultrastructural characterization of the endothelial cell in the rat liver sinusoid under normal and various experimental conditions, as a contribution to the distinction between endothelial and Kupffer cells. J Ultrastruct Res 1972; 38: 528-562.

25 Wisse E. Observations on the fine structure and peroxidase cytochemistry of normal rat liver Kupffer cells. J Ultrastruct Res 1974; 46: 393-426.

$26 \mathrm{Kmiec}$ Z. Cooperation of liver cells in health and disease. Adv Anat Embryol Cell Biol 2001; 161: III-XIII, 1-151.

27 Crispe IN. Liver antigen-presenting cells. J Hepatol 2011; 54: 357365.

28 Diehl L, Schurich A, Grochtmann R, Hegenbarth S, Chen L, Knolle PA. Tolerogenic maturation of liver sinusoidal endothelial cells promotes B7-homolog 1-dependent CD8 + T cell tolerance. Hepatology 2008; 47: 296-305.

29 Doherty DG, O'Farrelly C. Innate and adaptive lymphoid cells in the human liver. Immunol Rev 2000; 174: 5-20.

30 Herberman RB, Nunn ME, Lavrin DH. Natural cytotoxic reactivity of mouse lymphoid cells against syngeneic acid allogeneic tumors. I. Distribution of reactivity and specificity. Int J Cancer 1975; 16 : 216-229.

31 Kiessling R, Klein E, Wigzell H. "Natural" killer cells in the mouse. I. Cytotoxic cells with specificity for mouse Moloney leukemia cells. Specificity and distribution according to genotype. Eur J Immunol 1975; 5: 112-117.

32 Ferlazzo G, Morandi B. Cross-talks between natural killer cells and distinct subsets of dendritic cells. Front Immunol 2014; 5: 159.

33 Marcus A, Raulet DH. Evidence for natural killer cell memory. Curr Biol 2013; 23: R817-820.

34 Lanier LL. NK cell recognition. Annu Rev Immunol 2005; 23: 225 274.

35 Narni-Mancinelli E, Chaix J, Fenis A, Kerdiles YM, Yessaad N, Reynders $A$ et al. Fate mapping analysis of lymphoid cells expressing the NKp46 cell surface receptor. Proc Natl Acad Sci U S A 2011; 108: 18324-18329.

36 Elliott JM, Yokoyama WM. Unifying concepts of MHC-dependent natural killer cell education. Trends Immunol 2011; 32: 364-372.

37 Kim S, lizuka K, Kang HS, Dokun A, French AR, Greco S et al. In vivo developmental stages in murine natural killer cell maturation. Nat Immunol 2002; 3: 523-528.

38 Rodewald HR, Moingeon P, Lucich JL, Dosiou C, Lopez P, Reinherz EL. A population of early fetal thymocytes expressing Fc gamma RII/ III contains precursors of T lymphocytes and natural killer cells. Cell 1992; 69: 139-150.

39 Sojka DK, Plougastel-Douglas B, Yang L, Pak-Wittel MA, Artyomov MN, Ivanova $Y$ et al. Tissue-resident natural killer (NK) cells are cell lineages distinct from thymic and conventional splenic NK cells. Elife 2014; 3: e01659.

40 Vosshenrich CA, Garcia-Ojeda ME, Samson-Villeger SI, Pasqualetto $\checkmark$, Enault L, Richard-Le Goff $O$ et al. A thymic pathway of mouse natural killer cell development characterized by expression of GATA-3 and CD127. Nat Immunol 2006; 7: 1217-1224.

41 Di Santo JP. Natural killer cells: diversity in search of a niche. Nat Immunol 2008; 9: 473-475.

42 Wisse E, van't Noordende JM, van der Meulen J, Daems WT. The pit cell: description of a new type of cell occurring in rat liver sinusoids and peripheral blood. Cell Tissue Res 1976; 173: 423-435.

43 Sojka DK, Tian Z, Yokoyama WM. Tissue-resident natural killer cells and their potential diversity. Semin Immunol 2014; 26: 127-131.

44 Kaneda K, Wake K. Distribution and morphological characteristics of the pit cells in the liver of the rat. Cell Tissue Res 1983; 233: 485-505.

45 Bouwens L, Remels L, Baekeland M, Van Bossuyt H, Wisse E. Large granular lymphocytes or "pit cells" from rat liver: isolation, ultrastructural characterization and natural killer activity. Eur J Immunol 1987; 17: 37-42.

46 Bouwens L, Wisse E. Tissue localization and kinetics of pit cells or large granule lymphocytes in the liver of rats treated with biological response modifiers. Hepatology 1988; 8: 46-52. 
47 Bouwens L, Marinelli A, Kuppen PJ, Eggermont AM, van de Velde CJ, Wisse E. Electron microscopic observations on the accumulation of large granular lymphocytes (pit cells) and Kupffer cells in the liver of rats treated with continuous infusion of interleukin-2. Hepatology 1990; 12: 1365-1370.

48 Vanderkerken K, Bouwens L, Van Rooijen N, Van den Berg K, Baekeland $\mathrm{M}$, Wisse $\mathrm{E}$. The role of Kupffer cells in the differentiation process of hepatic natural killer cells. Hepatology 1995; 22: 283-290.

49 Vanderkerken K, Bouwens L, Wisse E. Characterization of a phenotypically and functionally distinct subset of large granular lymphocytes (pit cells) in rat liver sinusoids. Hepatology 1990; 12: 70-75.

50 Luo D, Vanderkerken K, Bouwens L, Kuppen PJ, Crabbe E, Wisse E. The number and distribution of hepatic natural killer cells (pit cells) in normal rat liver: an immunohistochemical study. Hepatology 1995; 21: 1690-1694.

51 Vermijlen D, Luo D, Robaye B, Seynaeve C, Baekeland M, Wisse E. Pit cells (Hepatic natural killer cells) of the rat induce apoptosis in colon carcinoma cells by the perforin/granzyme pathway. Hepatology 1999; 29: 51-56.

52 Wisse E, Braet F, Duimel H, Vreuls C, Koek G, Olde Damink SW et al. Fixation methods for electron microscopy of human and other liver. World J Gastroenterol 2010; 16: 2851-2866.

53 Vreuls C, Wisse E, Duimel H, Stevens K, Verheyen F, Braet F et al. Jetfixation: a novel method to improve microscopy of human liver needle biopsies. Hepatology 2014; 59: 737-739.

54 Gordon SM, Chaix J, Rupp LJ, Wu J, Madera S, Sun JC et al. The transcription factors T-bet and Eomes control key checkpoints of natural killer cell maturation. Immunity 2012; 36: 55-67.

55 Wang J, Li F, Zheng M, Sun R, Wei H, Tian Z. Lung natural killer cells in mice: phenotype and response to respiratory infection. Immunology 2012; 137: 37-47.

56 Rosmaraki EE, Douagi I, Roth C, Colucci F, Cumano A, Di Santo JP. Identification of committed NK cell progenitors in adult murine bone marrow. Eur J Immunol 2001; 31: 1900-1909.

57 Daussy C, Faure F, Mayol K, Viel S, Gasteiger G, Charrier E et al. T-bet and Eomes instruct the development of two distinct natural killer cell lineages in the liver and in the bone marrow. J Exp Med 2014; 211: 563-577.

58 O'Leary JG, Goodarzi M, Drayton DL, von Andrian UH. T cell- and B cell-independent adaptive immunity mediated by natural killer cells. Nat Immunol 2006; 7: 50-516.

59 Paust S, Gill HS, Wang BZ, Flynn MP, Moseman EA, Senman B et al. Critical role for the chemokine receptor CXCR6 in NK cell-mediated antigen-specific memory of haptens and viruses. Nat Immunol 2010; 11: 1127-1135.

60 Rouzaire P, Luci C, Blasco E, Bienvenu J, Walzer T, Nicolas JF et al. Natural killer cells and $T$ cells induce different types of skin reactions during recall responses to haptens. Eur J Immunol 2012; 42: 80-88.

61 Sun JC, Beilke JN, Lanier LL. Adaptive immune features of natural killer cells. Nature 2009; 457: 557-561.

62 Gillard GO, Bivas-Benita M, Hovav AH, Grandpre LE, Panas MW, Seaman MS et al. Thy $1+$ NK [corrected] cells from vaccinia virusprimed mice confer protection against vaccinia virus challenge in the absence of adaptive lymphocytes. PLoS Pathog 2011; 7: e1002141.

63 Cooper MA, Elliott JM, Keyel PA, Yang L, Carrero JA, Yokoyama WM. Cytokine-induced memory-like natural killer cells. Proc Natl Acad Sci U S A 2009; 106: 1915-1919.

64 Cunningham EC, Sharland AF, Bishop GA. Liver transplant tolerance and its application to the clinic: can we exploit the high dose effect? Clin Dev Immunol 2013; 2013: 419692.

65 Tiegs G, Lohse AW. Immune tolerance: what is unique about the liver. J Autoimmun 2010; 34: 1-6.

66 Protzer U, Maini MK, Knolle PA. Living in the liver: hepatic infections. Nat Rev Immunol 2012; 12: 201-213.

67 Dong Z, Wei H, Sun R, Hu Z, Gao B, Tian Z. Involvement of natural killer cells in Polyl:C-induced liver injury. J Hepatol 2004; 41: 966-973.

68 Hou X, Zhou R, Wei H, Sun R, Tian Z. NKG2D-retinoic acid early inducible-1 recognition between natural killer cells and Kupffer cells in a novel murine natural killer cell-dependent fulminant hepatitis. Hepatology 2009; 49: 940-949.
69 Zou Y, Chen T, Han M, Wang H, Yan W, Song G et al. Increased killing of liver NK cells by Fas/Fas ligand and NKG2D/NKG2D ligand contributes to hepatocyte necrosis in virus-induced liver failure. $\mathrm{J}$ Immunol 2010; 184: 466-475.

70 Jinushi M, Takehara T, Tatsumi T et al. Negative regulation of NK cell activities by inhibitory receptor CD94/NKG2A leads to altered NK cell-induced modulation of dendritic cell functions in chronic hepatitis C virus infection. J Immunol 2004;173: 6072-6081.

71 Jinushi M, Takehara T, Tatsumi T, Kanto T, Miyagi T, Suzuki T et al. Natural killer cell and hepatic cell interaction via NKG2A leads to dendritic cell-mediated induction of CD4 CD25 T cells with PD-1dependent regulatory activities. Immunology 2007; 120: 73-82.

72 Li F, Wei H, Gao Y, Gao Y, Xu L, Yin W et al. Blocking the natural killer cell inhibitory receptor NKG2A increases activity of human natural killer cells and clears hepatitis B virus infection in mice. Gastroenterology 2013; 144: 392-401.

73 Sun C, Fu B, Gao Y, Liao X, Sun R, Tian Z et al. TGF-betal downregulation of NKG2D/DAP10 and 2B4/SAP expression on human NK cells contributes to HBV persistence. PLoS Pathog 2012; 8: e1002594.

74 Lassen MG, Lukens JR, Dolina JS, Brown MG, Hahn YS. Intrahepatic IL-10 maintains NKG2A+Ly49- liver NK cells in a functionally hyporesponsive state. J Immunol 2010; 184: 2693-2701.

75 Gao B, Radaeva S. Natural killer and natural killer T cells in liver fibrosis. Biochim Biophys Acta 2013; 1832: 1061-1069.

76 Gao B, Radaeva S, Park O. Liver natural killer and natural killer T cells: immunobiology and emerging roles in liver diseases. J Leukoc Biol 2009;86: 513-528.

77 Radaeva S, Sun R, Jaruga B, Nguyen VT, Tian Z, Gao B. Natural killer cells ameliorate liver fibrosis by killing activated stellate cells in NKG2D-dependent and tumor necrosis factor-related apoptosisinducing ligand-dependent manners. Gastroenterology 2006; 130: 435-452.

78 Melhem A, Muhanna N, Bishara A, Alvarez CE, Ilan Y, Bishara T et al. Anti-fibrotic activity of NK cells in experimental liver injury through killing of activated HSC. J Hepatol 2006; 45: 60-71.

79 Muhanna N, Abu Tair L, Doron S, Amer J, Azzeh M, Mahamid M et al. Amelioration of hepatic fibrosis by NK cell activation. Gut 2011;60: 90-98.

80 Gur C, Doron S, Kfir-Erenfeld S, Horwitz E, Abu-Tair L, Safadi R et al. NKp46-mediated killing of human and mouse hepatic stellate cells attenuates liver fibrosis. Gut 2012; 61: 885-893.

81 Glassner A, Eisenhardt M, Kramer B, Körner C, Coenen M, Sauerbruch $\mathrm{T}$ et al. NK cells from HCV-infected patients effectively induce apoptosis of activated primary human hepatic stellate cells in a TRAIL-, FasL- and NKG2D-dependent manner. Lab Invest 2012; 92: 967-977.

82 Langhans B, Alwan AW, Kramer B, Glässner A, Lutz P, Strassburg CP et al. Regulatory CD4+ T cells modulate the interaction between NK cells and hepatic stellate cells by acting on either cell type. J Hepatol 2015; 62: 398-404.

83 Tamura F, Masuhara A, Sakaida I, Fukumoto E, Nakamura T, Okita K. FK506 promotes liver regeneration by suppressing natural killer cell activity. J Gastroenterol Hepatol 1998; 13: 703-708.

84 Sun R, Gao B. Negative regulation of liver regeneration by innate immunity (natural killer cells/interferon-gamma). Gastroenterology 2004; 127: 1525-1539.

85 Bi J, Zheng X, Chen Y, Wei H, Sun R, Tian Z. TIGIT safeguards liver regeneration through regulating natural killer cell-hepatocyte crosstalk. Hepatology 2014; 60: 1389-1398.

(c) (i) (\$) $\odot$ This work is licensed under a Creative Commons Attribution-NonCommercial-NoDerivs 4.0 International License. The images or other third party material in this article are included in the article's Creative Commons license, unless indicated otherwise in the credit line; if the material is not included under the Creative Commons license, users will need to obtain permission from the license holder to reproduce the material. To view a copy of this license, visit http://creativecommons.org/licenses/by-nc-nd/4.0/ 